\title{
Radiation Patterns From Plasma Enclosed Cylindrical Hypersonic Vehicles*
}

\author{
J. H. Harris, A. T. Villeneuve, and L. A. Broca ${ }^{1}$ \\ Antenna Department, Aerospace Group, Hughes Aircraft Company, Culver City, Calif.
}

(Received March 17. 1965; revised May 26, 1965)

\begin{abstract}
The effect of plasma sheaths on the radiation patterns of antennas on hypersonic vehicles is theoretically investigated. A simplified mathematical model is employed that consists of an infinitesimal longitudinal magnetic dipole on a plasma-enclosed cylinder. Computed radiation patterns for the point source and for a horn and a slotted array are presented.

The effects of varying both the permittivity and the loss tangent of the sheath are considered. It is found that in the lossless case the radiated power of an otherwise omnidirectional source is greatly reduced in spatial regions beyond the critical angles of the sheath as defined from geometrical optics. Also, the transmission loss at broadside due to a lossy sheath is found to be generally greater than predicted by plane-wave, plane-sheath approximations.

These results are extensions of earlier work by Harris. Some of the curves in this paper are corrections to the curves in the earlier work.
\end{abstract}

\section{Introduction}

The presence of ionized sheaths about hypersonic re-entry vehicles may have profound effects on the characteristics of electromagnetic waves radiated from or incident upon such vehicles. Effects such as radiation pattern distortion, antenna impedance changes, and signal attenuation may result from the ionized environment. The magnitudes of these effects depend in extremely complicated ways on a combination of such physical factors as vehicle shape and trajectory, frequency of the electromagnetic waves, composition of the atmosphere, etc. It becomes important, therefore, to attempt to determine the nature of some of these effects by representing the actual physical configurations by simplified mathematical models which are as simple as possible and yet can lead to a prediction of the salient features of the phenomena of interest.

This paper is devoted to a study of radiation pattern distortion which may result when cylindrical vehicles are surrounded by plasma sheaths. Since the primary interest is the ability to communicate through the plasma, it has been assumed that the radio frequency is above the plasma frequency for most cases considered. The effects of varying both the relative permittivity and loss tangent of the sheath are determined.

The results reported here are an extension of earlier work [Harris, 1963]. Radiation patterns in the presence of dissipative sheaths and in regions beyond the critical angles which result from the presence of a

\footnotetext{
*This work was sponsored by the Air Force Cambridge Research Laboratories, Office of Aerospace Research, under Contract No. AF 19(604)-8386.

${ }^{1}$ Presently employed by Westinghouse Electric Corporation, Defense and Space Center. Advanced Development Engineering, 487-L Group, Baltimore, Md.
}

sheath are important additions to this earlier work. In addition, the patterns between broadside and the critical angle for the lossless plasmas shown in the present study are corrections to the patterns included in the previous paper.

\section{Presentation of the Problem}

\subsection{Mathematical Model}

In this paper an exact solution for the radiation field of an antenna in an idealized geometry is presented. The geometry is a model for the problem of an antenna mounted on the side of a long slender vehicle. Depicted in figure 1 , the model consists of a finite magnetic line source flush-mounted on an infinitely long, perfectly conducting cylinder. The cylinder is surrounded by a concentric but not contiguous cylindrical plasma sheath. This model incorporates some of the more salient features of the true problem - the physical characteristics of the long slender vehicle, the sheath, and the gap between the sheath and the vehicle. The low density region beyond the sheath is neglected because the propagation frequency is assumed to be comparable to the plasma frequency in the high density portion of the sheath and therefore much higher than the plasma frequency in the low density region.

The approximation of the actual sheath by a cylindrical sheath with homogeneous properties appears to be reasonable, based on approximate solutions for electron concentration about re-entry vehicles and on the models considered by other investigators [e.g., Rotman and Meltz, 1961; Swift, 1964]. The assumption of infinite lengths, which allows an exact solution 


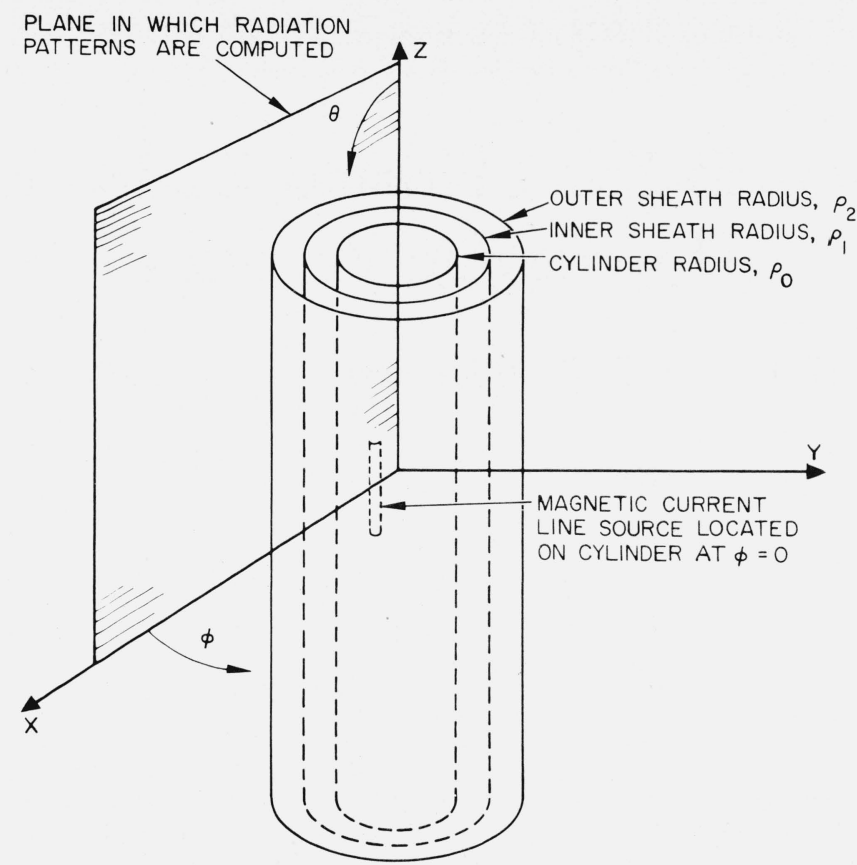

Figure 1. Geometrical configuration for a line source on a conducting cylinder surrounded by a plasma sheath.

to be obtained, also appears reasonable for the case of longitudinal slots, so long as the vehicle and the sheath are long compared with their diameters.

In short, this paper deals with the exact solution of a physically meaningful problem. Radiation fields for the magnetic line source on the cylinder are computed as a function of $\theta$ in the plane that contains the line source and the cylinder axis. Parameters which affect the radiation are the three radii-the cylinder radius and the radii of the inner and outer bounderies of the sheath (respectively denoted by $\rho_{0}, \rho_{1}$, and $\rho_{2}$ in the text)-and the electron density, and collision frequency in the sheath. The d-c magnetic effects due to the earth's field or any magnetic devices that may be in the vehicle are neglected.

\subsection{Selection of Parameter Values}

The relations between the collision and plasma frequencies and the sheath constitutive parameters are [Allis, Buchsbaum, and Bers, 1963]

$$
\nu=\omega\left(\frac{\sigma / \omega \epsilon_{0}}{1-\epsilon / \epsilon_{0}}\right)
$$

and

$$
\omega_{p}=\omega\left(1-\epsilon / \epsilon_{0}\right)^{-1 / 2}\left[\left(1-\epsilon / \epsilon_{0}\right)^{2}+\left(\frac{\sigma}{\omega \epsilon_{0}}\right)^{2}\right]^{1 / 2}
$$

where $\omega$ is the propagation angular frequency, $\nu$ is the collision frequency, and $\omega_{p}$ is the plasma frequency. Table 1 has been prepared to indicate the various
TABLE 1. Ratio of collision and plasma frequencies to propagation frequencies for the sheath constitutive parameters of figures 2

\begin{tabular}{|c|c|c|c|c|c|c|c|c|c|}
\hline \multirow{2}{*}{$\begin{array}{l}\text { Fre- } \\
\text { quency } \\
\text { ratio }\end{array}$} & \multicolumn{9}{|c|}{ Relative permittivity, $\epsilon / \epsilon_{0}$} \\
\hline & 0.20 & & & 0.40 & & & & 0.80 & \\
\hline $\begin{array}{l}\sigma / \omega \epsilon_{0} \\
\nu / \omega \\
\omega_{p} / \omega\end{array}$ & $\begin{array}{l}0 \\
0 \\
0.894\end{array}$ & $\begin{array}{l}0 \\
0 \\
0.775\end{array}$ & $\begin{array}{c}0.01 \\
.0167 \\
.777\end{array}$ & $\begin{array}{l}0.05 \\
.0834 \\
.779\end{array}$ & $\begin{array}{c}0.10 \\
.167 \\
.786\end{array}$ & $\begin{array}{l}0.50 \\
0.834 \\
1.008\end{array}$ & $\begin{array}{l}0 \\
0 \\
0.401\end{array}$ & $\begin{array}{l}0.05 \\
.25 \\
.414\end{array}$ & $\begin{array}{r}0.10 \\
.50 \\
.448\end{array}$ \\
\hline
\end{tabular}
and 3.

values of $\nu$ and $\omega_{p}$ in the sheath for figures 2 and 3 as computed from (1) and (2). It should be noted that for the most lossy case, $\sigma / \omega \epsilon_{0}=0.50$, the plasma frequency is greater than the propagation frequency, but because of the high collision frequency, the relative permittivity, $\epsilon / \epsilon_{0}=0.40$, remains greater than zero. ${ }^{2}$

The mathematical technique used in determining the radiation patterns was taken from Wait [1959] and is described in detail in the earlier paper [Harris, 1963]. The field expression that yields the radiation field in the plane of interest ${ }^{3}$ is

$$
E_{\phi}=\left.\left(2 i \omega \mu_{0} \frac{e^{-i k R}}{R}\right) \sum_{m=-\infty}^{\infty} \sqrt{k_{0}^{2}-h^{2}} b_{m}(h) e^{i m \pi / 2}\right|_{h=k_{0} \cos \theta}
$$

\footnotetext{
Some relationships between flight conditions and parameter values are discussed in the report on which this paper is based. [Harris, Villeneuve, and Broca, 1964].

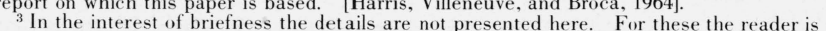
referred to Harris [1963] and to Harris, Villeneuve, and Broca, [1964].
} 

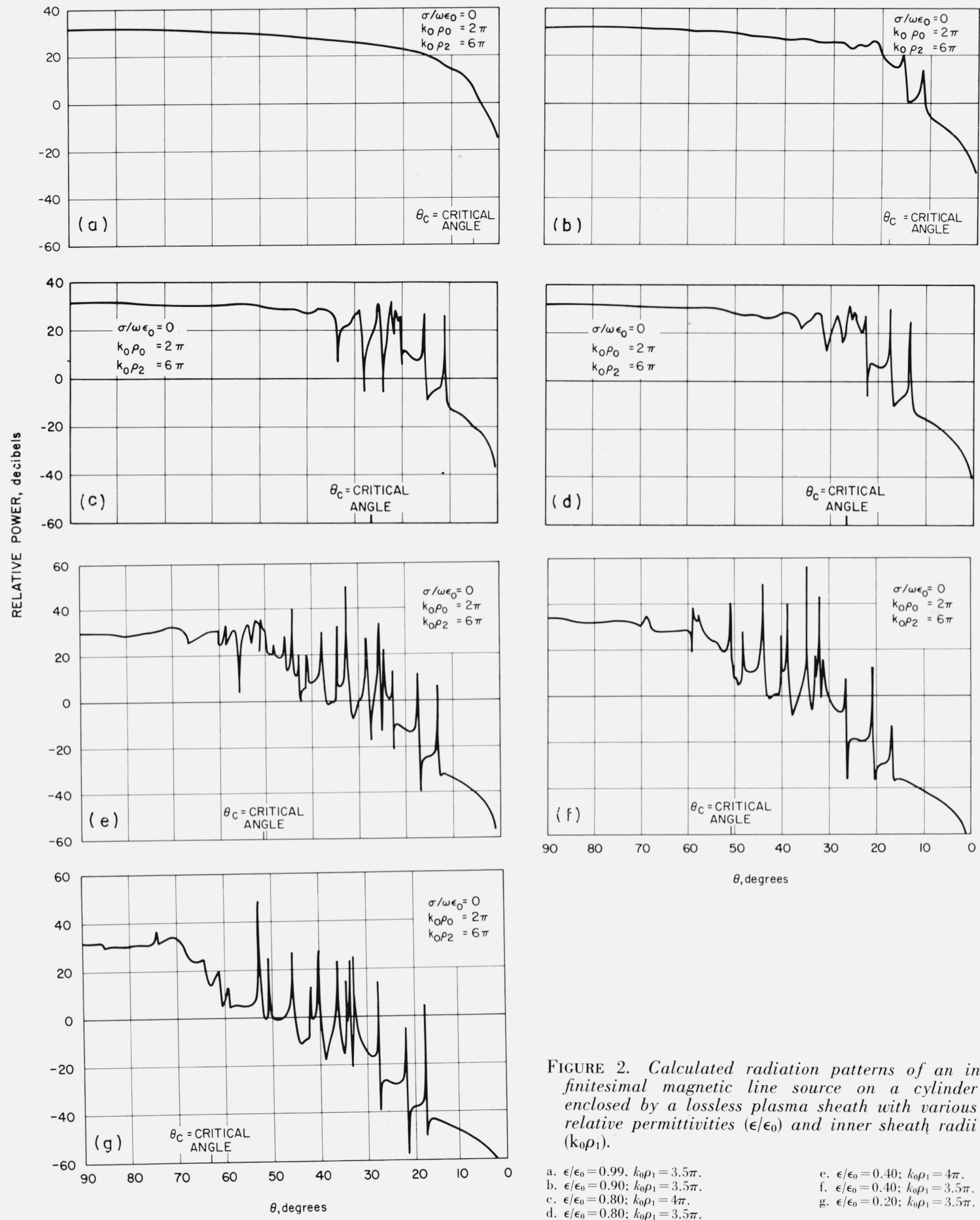

FIGURE 2. Calculated radiation patterns of an infinitesimal magnetic line source on a cylinder enclosed by a lossless plasma sheath with various relative permittivities $\left(\epsilon / \epsilon_{0}\right)$ and inner sheath radii $\left(\mathrm{k}_{0} \rho_{1}\right)$.
a. $\epsilon / \epsilon_{0}=0.99, k_{0} \rho_{1}=3.5 \pi$.
b. $\epsilon / \epsilon_{0}=0.90: k_{0} \rho_{1}=3.5 \pi$.
c. $\epsilon / \epsilon_{0}=0.80 ; k_{0} \rho_{1}=4 \pi$.
e. $\epsilon / \epsilon_{0}=0.40 ; k_{0} \rho_{1}=4 \pi$.
f. $\epsilon / \epsilon_{0}=0.40 ; k_{0} \rho_{1}=3.5 \pi$
d. $\epsilon / \epsilon_{0}=0.80 ; k_{0} \rho_{1}=3.5 \pi$
g. $\epsilon / \epsilon_{0}=0$. 

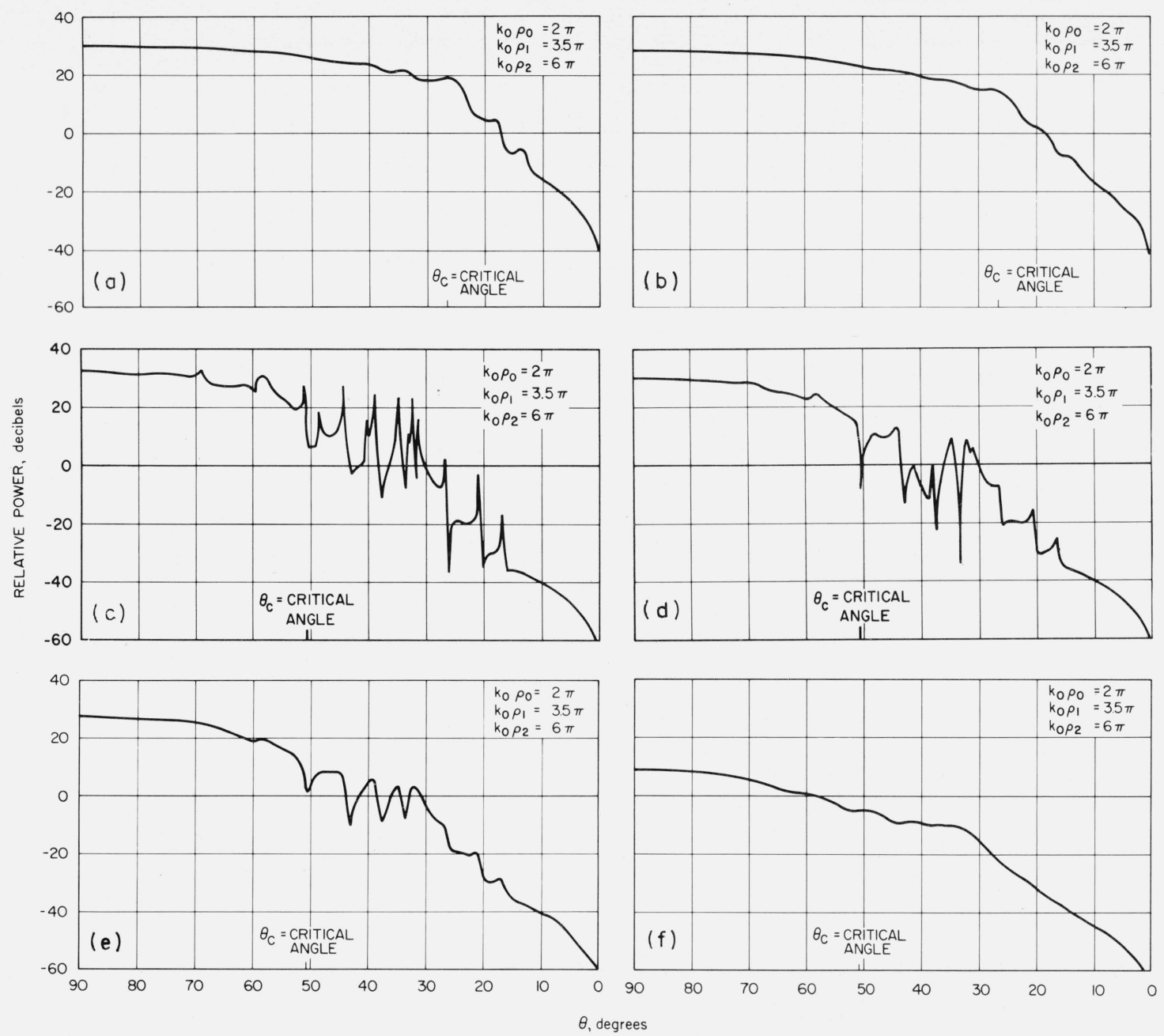

FIGURE 3. Calculated radiation patterns of an infinitesimal magnetic line source on a cylinder enclosed by a lossy plasma sheath with various relative permittivities $\left(\epsilon / \epsilon_{0}\right)$ and normalized conductivities $\left(\sigma / \omega \epsilon_{0}\right)$.

a. $\epsilon / \epsilon_{0}=0.80 ; \sigma / \omega \epsilon_{0}=0.05$
b. $\epsilon / \epsilon_{0}=0.80 ; \sigma / \omega \epsilon_{0}=0.10$

c. $\epsilon / \epsilon_{0}=0.40 ; \sigma / \omega \epsilon_{0}=0.01$. d. $\epsilon / \epsilon_{0}=0.40 ; \sigma / \omega \epsilon_{0}=0.05$

e. $\epsilon / \epsilon_{0}=0.40 ; \sigma / \omega \epsilon_{0}=0.10$

f. $\epsilon / \epsilon_{0}=0.40 ; \sigma / \omega \epsilon_{0}=0.50$ where $R$ and $\theta$ are the usual spherical coordinates measured from the same origin as the cylindrical coordinates. The effect of the sheath and cylinder is entirely represented in the term $b_{m}(h)$ which may be written

$$
b_{m}(h)=\left[\int_{-\infty}^{\infty} d z E_{\text {aperture }} e^{i h z}\right] f_{m}(h)
$$

The integral term in (4) contains the antenna element and array factors, while the term $f_{m}(h)$, which is a function of the geometry, enters into the cylinder factor. This cylinder factor is of principal interest, and is discussed in section 3 but is not presented explicitly because of its excessive length. The total solution is discussed in section 4 .

\section{Radiation Patterns}

In this section the computed radiation field in the plane of the magnetic line source and the cylinder axis is presented. The strength of the source is maintained constant for all cases, and the curves represent the field of an infinitesimal magnetic dipole of fixed dipole moment on the cylinder. Each of the curves in figures 2 and 3 may thus be compared directly. The field is termed the "cylinder factor" and is exclusive of the element and array factors.

\subsection{Lossless Sheath}

A lossless sheath is one in which the conductivity is zero. That is, the wave number in the sheath is 
real and given by

$$
k=k_{0} \sqrt{\epsilon / \epsilon_{0}}
$$

indicating that there are no heat losses in the sheath. The radiation patterns of a unit magnetic source in the presence of such a sheath are shown in figures $2 \mathrm{a}$ through $2 \mathrm{~g}$. The dielectric constant in the sheath and the sheath thickness are parameters for the curves. The critical angle $\theta_{c}$ shown on the patterns is defined by

$$
\sin ^{2} \theta_{c}=\epsilon / \epsilon_{0}
$$

The values $k_{0} \rho_{0}=2 \pi$ and $k_{0} \rho_{2}=6 \pi$ are constant and correspond, respectively, to a cylinder radius of 1 free space wavelength and an outer sheath radius of 3 free space wavelengths. The values of $3.5 \pi$ and $4 \pi$ for $k_{0} \rho_{1}$, the inner sheath radius, correspond respectively to 1.75 and 2 free space wavelengths.

The $\epsilon / \epsilon_{0}=0.99$ case (fig. 2a) is essentially the sheathfree case and exhibits none of the amplitude fluctuations which are characteristic of the radiation in the presence of denser sheaths. The case $\epsilon / \epsilon_{0}=0.90$ (fig. $2 \mathrm{~b}$ ) corresponds to a ratio of plasma frequency to propagation frequency of 0.316 and is somewhat more characteristic of sheath effects. Three fairly modest peaks appear between 10 and $22^{\circ}$, or in the vicinity of the critical angle which is at $18^{\circ}$ in this case. The power drops to more than $20 \mathrm{~dB}$ below broadside power at about $15^{\circ}$, excluding the peak at $11^{\circ}$, while in the $\epsilon / \epsilon_{0}=0.99$ case it drops to this cutoff value at $9^{\circ}$.

The $\epsilon / \epsilon_{0}=0.80$ cases are shown in figures $2 \mathrm{c}$ and $2 d$. These correspond to sheath thicknesses $\left(\rho_{2}-\rho_{1}\right)$ of 1 and 1.25 free space wavelengths, respectively. The latter case has rather sharp cutoff at about $22.5^{\circ}$ and a rather interesting narrow beam at $13.5^{\circ}$ of approximate width $1^{\circ}$ and magnitude $26 \mathrm{~dB}$. Such a beam is accounted for physically as the radiation field of a leaky wave which, due to leakage of energy as it propagates, is attenuated slowly along the cylindrical structure. ${ }^{4}$ The pattern for the one-wavelength-thick sheath exhibits deeper nulls than that for the 1.25-wavelength sheath. While the pattern essentially remains above the $-20 \mathrm{~dB}$ level from 90 to about $20.5^{\circ}$, signal levels fall below the $-20 \mathrm{~dB}$ level over $1^{\circ}$ widths at angles as great as $33^{\circ}$.

The $\epsilon / \epsilon_{0}=0.4$ cases (figs. $2 \mathrm{e}$ and $2 \mathrm{f}$ ) exhibit a great number of peaks and valleys. Of interest here, in addition to the narrow beams, is the great difference in the patterns caused by the $1 / 4$-wavelength change in sheath thickness. Even the level at broadside varies by $4 \mathrm{~dB}$ in the two cases, and while the deviations from a smooth curve occur at about the same angles, these deviations generally differ in form in the two cases. The pattern essentially drops below $-20 \mathrm{~dB}$ at $40.5^{\circ}$ in the 1.25 -wavelength case and at $42.5^{\circ}$ in the l-wavelength case.

4 The relationship between leaky wave, i.e., nonspectral, modes associated with a structure and the characteristics of the radiation patterns from the structure is discussed in great detail in Tamir and Oliner [1962].
The final lossless case presented corresponds to $\epsilon / \epsilon_{0}=0.2$ (fig. $2 \mathrm{~g}$ ). Here the pattern essentially drops below $-20 \mathrm{~dB}$ at $61^{\circ}$, and once again a spectrum of narrow, high-intensity beams is present. It is evident that decreasing the sheath permittivity increases the number of significant leaky wave beams.

\subsection{Lossy Sheath}

\section{a. Pattern Characteristics}

A lossy sheath is one in which the conductivity is finite and nonzero. In this case the propagation constant in the sheath is given by

$$
k^{2}=k_{0}^{2}\left[\frac{\epsilon}{\epsilon_{0}}-i \frac{\sigma}{\omega \epsilon_{0}}\right]
$$

which implies that there is electromagnetic energy lost to the sheath in the form of heat due to collisions between the electrons and other particles in the sheath. The assumption made here is that the power absorption does not raise the temperature of the sheath sufficiently to alter the electron density. Figures 3a through $3 \mathrm{f}$ indicate the radiation patterns of an infinitesimal magnetic dipole on the cylinder in the presence of a sheath of thickness 1.25 free space wavelengths with relative permittivities $\epsilon / \epsilon_{0}=0.80$ and $\epsilon / \epsilon_{0}=0.40$ and with normalized conductivity $\sigma / \omega \epsilon_{0}$ as a parameter.

Figures $3 \mathrm{a}$ through $3 \mathrm{f}$, taken in conjunction with figures $2 \mathrm{~d}$ and $2 \mathrm{f}$, indicate a phenomenon that had been previously hypothesized: that the sharp peaks of the pattern in the lossless case would be flattened out as a result of increased conductivity. This predicted smoothing is apparent in the patterns. Indeed, the radiation field in the highest loss cases is virtually monotonic.

\section{b. Power Loss}

The radiation patterns shown in this section cannot be used directly to determine the power radiated by the antenna nor the power loss in the sheath since such calculations would require a knowledge of the antenna impedance [Villeneuve, 1965]. If the assumption is made, however, that the impedance seen by the antenna is essentially constant for fixed sheath thickness and permittivity, then the difference in the areas under the power curves for lossless and lossy sheaths represents the power lost in the sheath. Of perhaps greater interest is the reduction in transmitted power at broadside as the losses are increased, again under the assumption that the computed patterns for a given sheath thickness and permittivity represent the same constant power out of the antenna. Table 2 indicates this power loss at broadside for the cases of figures 3a through $3 \mathrm{f}$. Also included in this table for comparison is the power density transmitted through a plane sheet when a plane wave is normally 
TABLE 2. Broadside transmission loss through plasma sheath

\begin{tabular}{c|r|r|r|r|r}
\hline \hline \multirow{2}{*}{ Relative permittivity, $\epsilon / \epsilon_{0}$} & \multicolumn{5}{|c}{ Normalized conductivity, $\sigma / \omega \epsilon_{0}$} \\
\cline { 2 - 6 } & 0 & 0.01 & 0.05 & 0.10 & 0.50 \\
\hline 0.80 & & & & & \\
$\mathrm{~A}^{*}$ & 0 & - & -2.0 & -4.0 & - \\
$\mathrm{B}^{*}$ & 0 & - & -2.4 & -3.9 & - \\
0.40 & & & & & \\
$\mathrm{~A}^{*}$ & 0 & -0.80 & -3.6 & -6.4 & -24.8 \\
$\mathrm{~B}^{*}$ & 0 & -.30 & -0.67 & -3.7 & -22.6 \\
\hline
\end{tabular}

*A $=$ ratio of power transmitted in $\mathrm{dB}$ at broadside through a lossy cylindrical sheath to the power transmitted through a lossless cylindrical sheath of the same thickness $(1.25$ free space wavelengths) and the same permittivity.

${ }^{*} \mathrm{~B}=$ ratio of power density transmitted through a plane lossy sheath to the power trans. mitted through a plane lossless sheath of the same thickness ( 1.25 free space wave lengths) and permittivity when a plane wave is normally incident on the plane sheath.

incident. The thickness and the constitutive parameters of the plane sheath correspond to those same values in the cylinder problem. Powers are shown relative to the lossless sheath. It is intersting to note that the approximation of a plane wave propagating through a plane sheath, which is often used to estimate transmission loss, gives fairly comparable results with the transmission loss at broadside for the cylinder case and thus is somewhat justified as a first order approximation, for the cases considered here although the plane approximation is seen to generally yield a smaller loss than does the cylindrical geometry. Different conclusions have been reached by Swift [1964] for the overdense plasma and smaller values of $k_{0} \rho_{0}$. The plane wave approximation was computed from the following expression [Stratton, 1941]:

$$
\frac{E_{\text {transmitted }}}{E_{\text {incident }}}=\frac{4 e^{-i k_{0} d\left(k / k_{0}-1\right)}}{\left[2+\frac{k}{k_{0}}+\frac{k_{0}}{k}\right]\left[1-\left(\frac{k-k_{0}}{k+k_{0}}\right)^{2} e^{-i 2 k d}\right]}
$$

where $k$ is defined in (7), $k_{0}$ is the free space propagation constant, and $d$ is the thickness of the sheath.

\subsection{Comparison With Planar Radiation Problem}

As one method of checking on the reasonableness of the radiation patterns for the cylindrical case, the limiting case of a slot in a groundplane was considered. A plasma slab was located above the groundplane and separated from it as illustrated in figure 4 . On applying the boundary conditions at the groundplane and at the slab surfaces and on using the principle of stationary phase [Di Francia, 1955] to evaluate the radiation field, the following expression is obtained for $E_{\phi}$ in the plane $\phi=0$.

$$
E_{\phi}=4 \cos ^{2} \theta \frac{\sqrt{\epsilon_{2}-\sin ^{2} \theta}}{P}
$$

where $\theta$ is measured from broadside and $\epsilon_{2}$ is the slab relative permittivity. The denominator is given by

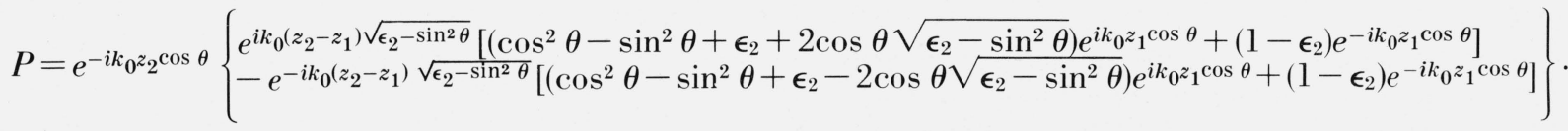

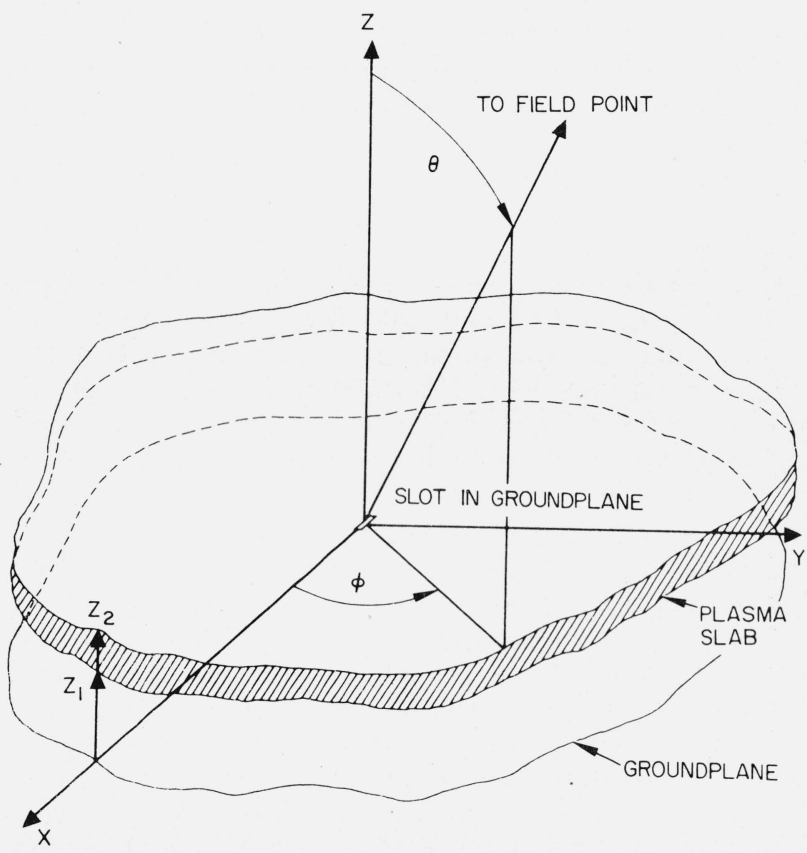

When $\sin ^{2} \theta$ is greater than $\epsilon_{2}$, the square root is negative imaginary. This expression for $E_{\phi}$ has been computed as a function of $\theta$ for the case of $\epsilon_{2}=0.20$, $k_{0} z_{1}=1.5 \pi$, and $k_{0} z_{2}=4 \pi$. This case corresponds to the cylindrical case for which $k_{0} \rho_{0}=2 \pi, k_{0} \rho_{1}=3.5 \pi$, and $k_{0} \rho_{2}=6 \pi$. The resulting curve normalized to its value at $\theta=0$ is shown in figure 5 . While this curve is much smoother than those for the corresponding cylindrical geometry of figure $2 \mathrm{~g}$, several very important characteristics are evident. First, most of the radiation is confined between broadside and the critical angle. Second, the peak which occurs near the critical angle corresponds to that type which occurs when there is no gap between the slab and the groundplane. [Tamir and Oliner, 1962]. Third, a high, very narrow peak occurs at about $\theta=57.9^{\circ}$. This peak results from the presence of the interface at $z_{1}$ and disappears as $z_{1}$ approaches zero. The value of the field at this angle, denoted by $\theta_{0}$, is given by

$$
\left|E_{\phi}\left(\theta_{0}\right)\right|=\cos \theta_{0} e^{k_{0}\left(z_{2}-z_{1}\right)} \sqrt{\sin ^{2} \theta_{0}-\epsilon_{2}}
$$

FIGURE 4. Slot in plasma-covered groundplane. 


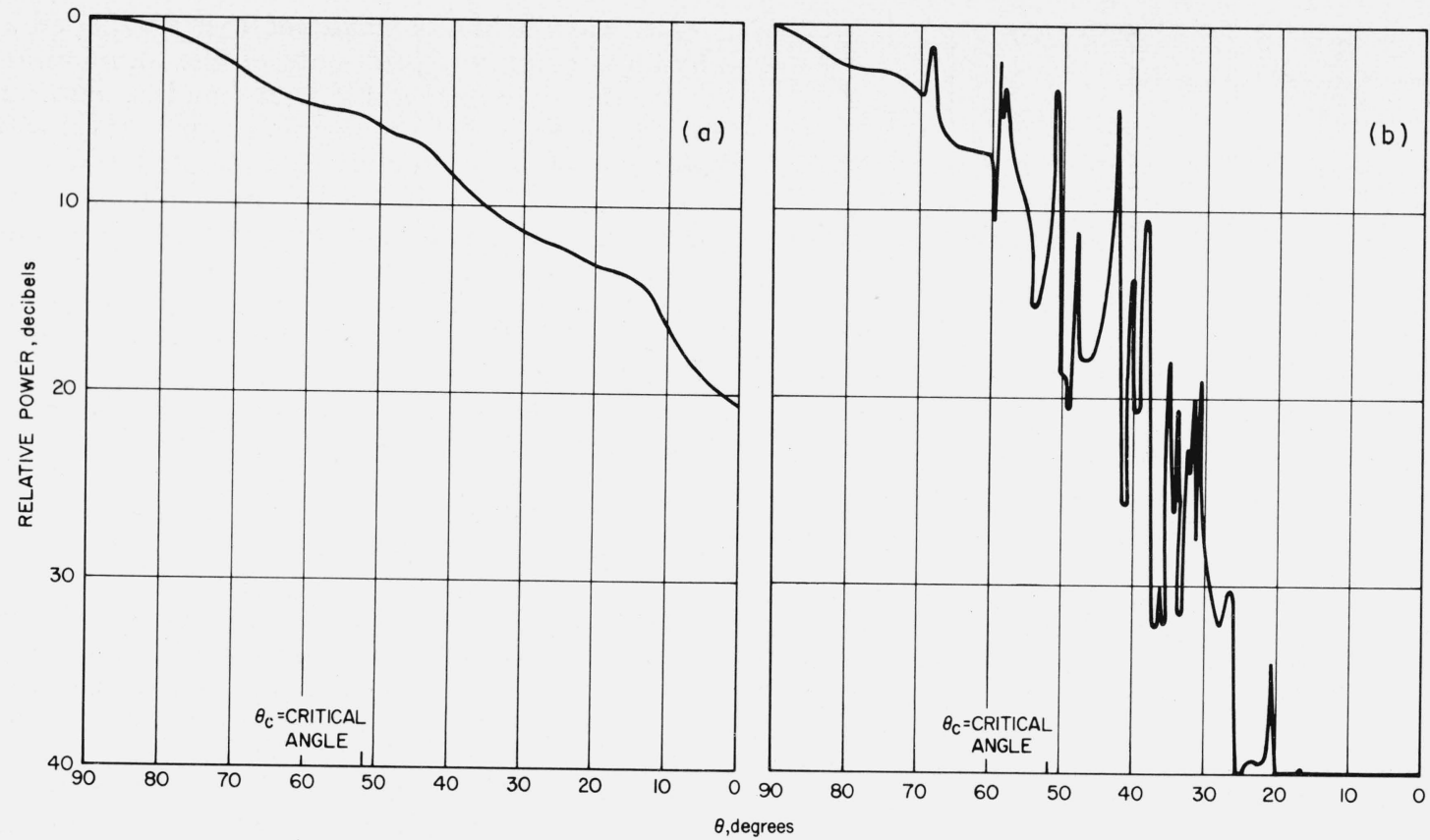

FIGURE 6. Radiation patterns of wide-beam horn.

a. Measured pattern of horn alone.
b. Calculated pattern of horn on cylinder enclosed by a lossless plasma sheath. $\epsilon / \epsilon_{0}=0.4, \sigma=0, k_{0} \rho_{0}=2 \pi, k_{0} \rho_{1}=3.5 \pi, k_{0} \rho_{2}=6 \pi$

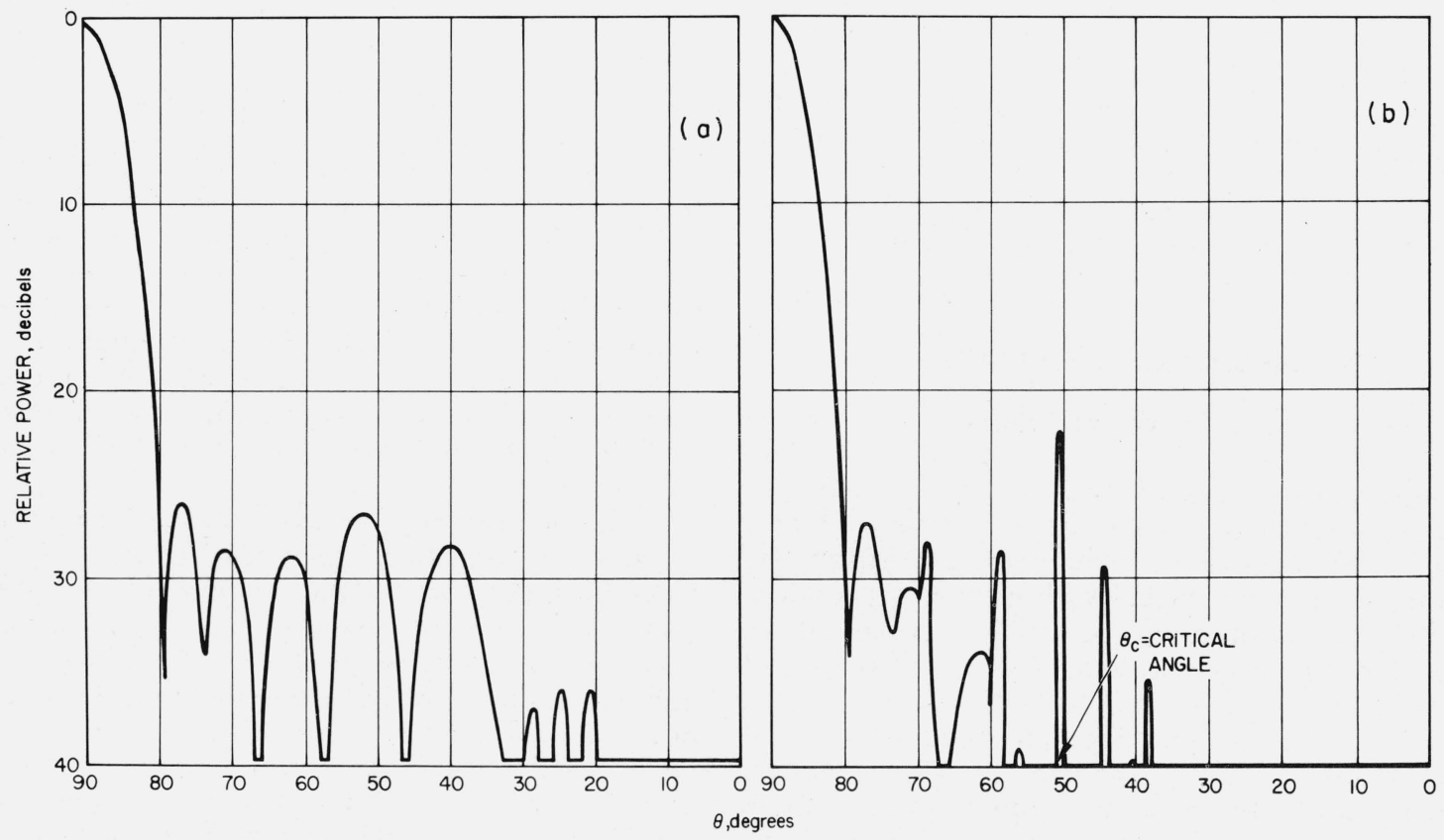

FiguRE 7. Radiation patterns of 20-element, 25-dB Tchebyscheff array.

a. Measured pattern of array alone.
b. Calculated pattern of array on a cylinder enclosed by a lossless plasma sheath. $\epsilon / \epsilon_{0}=0.4, \sigma=0, k_{0} \rho_{0}=2 \pi, k_{0} \rho_{1}=3.5 \pi, k_{0} \rho_{2}=6 \pi$. 
As an example, patterns for a horn antenna and a 20-element array are shown in figures 6 and 7; actual patterns measured in the absence of the cylinder and plasma are presented in figures $6 \mathrm{a}$ and $7 \mathrm{a}$, respectively, while the radiation pattern determined analytically for each type of antenna by multiplying the measured patterns by the cylinder factors is shown in figures $6 \mathrm{~b}$ and $7 \mathrm{~b}$. The horn and the narrow-beam array are assumed to be on a vehicle for which $\epsilon / \epsilon_{0}$ $=0.4, \sigma=0$, and $k_{0} \rho_{1}=3.5 \pi$ as in figure $2 \mathrm{f}$. The effect of the cylinder factor in the total patterns can be seen. The pattern of the narrow-beam array remains relatively unaffected by the plasma while that of the wide-beam horn is significantly tapered. (Only points at quarter-degree intervals were used in the computation of these composite patterns.)

When a vehicle is at intermediate altitudes, the loss factor due to increased collision frequency in the sheath plays an important role. Various loss factors for typical sheath conditions are shown in the rows marked "A" in table 2. As has been pointed out, the plane-wave plane-sheath approximation gives a general idea of the transmission loss through the sheath at broadside.

\section{Conclusions}

The principal results of this study are the numerous antenna patterns presented in the body of the report. They indicate the effect of plasma sheaths on the radiation patterns of antennas on hypersonic vehicles by an illustration of these effects on a simplified mathematical model. An important additional result is the establishment of a rule-of-thumb that the cylinder factor falls off rapidly beyond the critical angles.
The transmission loss through a lossy sheath at broadside is generally greater than the result predicted by the usual approximation of a plane wave at normal incidence on a plane sheath, although this approximation is useful in obtaining an estimate of the loss. The computations for this report (table 2) show a maximum difference in transmission loss of $3 \mathrm{~dB}$ between the cylindrical and the plane-wave values. Finally, there is the large number of narrow beams due to leaky wave poles which are generated by the sheath.

\section{References}

Allis, W. P., S. J. Buchsbaum, and A. Bers (1963), Waves in Anisotropic Plasmas, pp. 18-26 (MIT Press, Cambridge, Mass.).

di Francia, G. Toraldo (1955), Electromagnetic Waves, pp. 35-39 (Interscience Publishers, Inc., New York, N.Y.).

Harris, J. H. (1963), Radiation through cylindrical plasma sheaths, J. Res. NBS 67D (Radio Prop.), No. 6, 717-733.

Harris, J. H., A. T. Villeneuve, and L. A. Broca (1964), Radiation patterns of antennas on plasma enclosed cylinders, Report No. AFCRL 64-993, Air Force Cambridge Research Laboratories.

Rotman, W., and G. Meltz (Mar. 1961), Experimental investigation of the electromagnetic effects of reentry, AFCRL Document 87, Air Force Cambridge Research Laboratories, Bedford, Mass.

Stratton, J. A. (1941), Electromagnetic Theory, p. 512 (McGraw-Hill Book Co., Inc., New York, N.Y.).

Swift, C. T. (1964), Radiation patterns of a slotted cylinder antenna in the presence of an inhomogeneous lossy plasma, IEEE Trans. Ant. Prop. AP-1 2, No. 6, 728-738.

Tamir, T., and A. A. Oliner (1962), The influence of complex waves on the radiation field of a slot-excited plasma layer, IRE Trans. Ant. Prop. AP-10, No. 1, 55-65.

Villeneuve, A. T. (1965), Admittance of waveguide radiating into plasma environment, IEEE Trans. Ant. Prop. AP-13, No. 1, 115-121.

Wait, J. R. (1959), Electromagnetic Radiation from Cylindrical Structures (Pergamon Press, New York and London).

(Paper 69D10-567) 